\title{
A review of bone marrow examinations in Ibn Sena Teaching Hospital in Mosul
}

\author{
Mohammed H. Awaad \\ Department of Pathology, College of Medicine, University of Mosul, Mosul, Iraq. \\ Correspondence: Mohammed H. Awaad.mohammadh.awad@yahoo.com.
}

(Ann Coll Med Mosul 2018; 40 (2): 69-73).

Received: $2^{\text {nd }}$ Oct. 2013; Accepted: $19^{\text {th }}$ Mar. 2014.

\begin{abstract}
Objective: To assess the indications and different pathologies of bone marrow aspirates and to evaluate and analyze a considerable percentage of dry blood taps and normal bone marrow aspirates.

Methods: A retrospective study. All request procedures and reports of bone marrow examinations between January 2001 and December 2008 were reviewed.

Results: There were (2008) bone marrow aspirations performed during the period, $1258(63 \%)$ of the marrow aspirations were non-malignant and $750(37 \%)$ of the samples were found to be malignant. In 216 cases (11\%) marrow aspirates were found to be non-conclusive. There were $154(8 \%)$ full blood tap a recent full blood picture performed at the time of the bone marrow examination. The commonest indication was for investigation of thrombocytopenia and the most frequent diagnosis encountered was acute leukemia.

Conclusion: The bone marrow aspiration and trephine biopsy (scientific speaking) are complementary and give a higher diagnostic yield when both are available for a patient. This study also reveals that the failure rate of obtaining either of the samples is not significant and this issue needs to be addressed appropriately.
\end{abstract}

Keywords: Bone marrow aspiration, complete blood count.

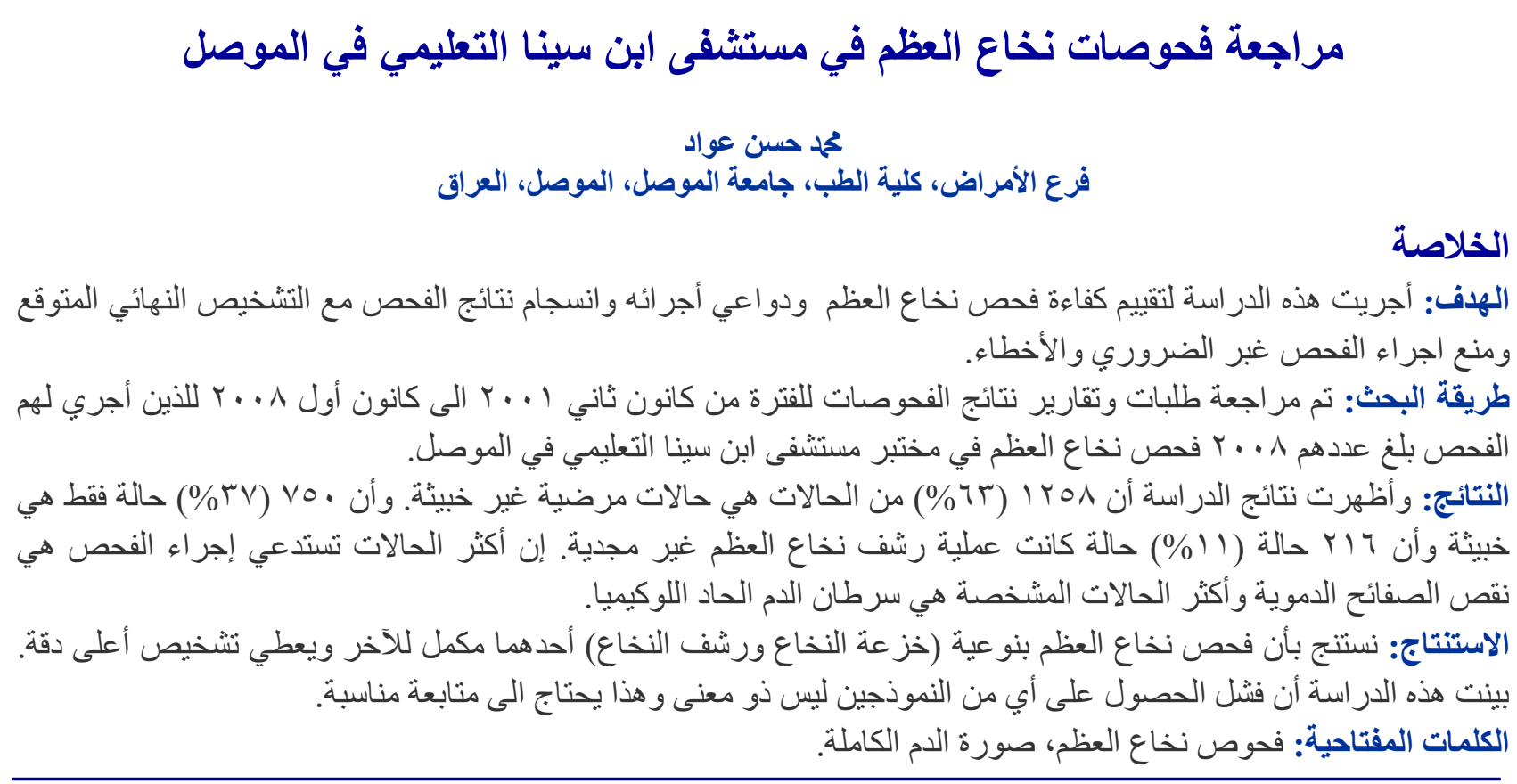

\section{INTRODUCTION}

$\mathrm{B}$ one marrow (BM) examination is a common practice in medicine. It is a key investigation in the diagnosis of many hematological and nonhematological disorders ${ }^{1}$. This evaluation is considered essential in patients with unexplained abnormality of any peripheral blood cell type. It has also become an important component as a followup evaluation of patients who undergo chemotherapy, bone marrow transplantation and other modalities of medical therapy. ${ }^{2}$ 
Basically there are two types of samples obtained for the examination, the bone marrow aspirate and trephine biopsy; the latter also known as a core biopsy.

In the first procedure, a small amount of liquid bone marrow is aspirated from the posterior iliac crest or sternum with a special needle. The aspirates are then used to make several thin smears on glass microscope slides. These smears are then stained with one of the Romanowsky stains such as May-Grunwald-Giemsa (MMG). ${ }^{3}$

The stained smears are primarily used to study the morphology and maturation stages of the blood cells. Such smears are also used to conduct differential counts as well as assessment of the myeloid to erythroid ratio. The iron store can also be assessed. In addition the marrow aspirate samples can be utilized for other tests such as flow cytometric immunophenotyping, cytogenetics and molecular studies.

Trephine biopsy on the other hand has an advantage in that it provides information on details of marrow architecture and cellularity that is lost in the marrow aspirate smear, thereby increases the chances of diagnosing focal lesions. Although it is not done in this center, In this process the sample is generally obtained from the posterior superior iliac crest where the marrow cavity is deep and there are no vital organs at risk, and the procedure is out of the sight of the patient. ${ }^{2}$ The sample is fixed, decalcified prior to paraffin embedding, and processed in the same manner as other surgical specimens. Hematoxylin and eosin (H \& E) is the routine stain used for the section and special stain can be employed if needed. The whole process usually takes about three days compared to the bone marrow aspirate smears which are usually completed on the same day.

It is generally recognized that optimal evaluation of the bone marrow involves examination of the trephine biopsy and aspirate material, obtained at the same time. Obtaining both specimens simultaneously is also cost effective in that it eliminates the need for the procedure to be repeated on the patient.

Absolute contraindications to obtaining bone marrow material are few. The primary contraindication is hemophilia and other hereditary bleeding tendencies although thrombocytopenia is not. $^{3}$
The two procedures discussed are generally safe but they are not entirely risk-free. ${ }^{4}$ There have been a number of reports on the morbidity and mortality resulting from these procedures ${ }^{5,6}$ Pain, bleeding and infections are recognized complications of aspirations at the posterior iliac crest, while death from cardiac tamponade has been reported, albeit rarely from sternal bone marrow aspiration. ${ }^{6}$ Therefore both procedures should be performed only when there is a clear clinical indication.

This paper presents a retrospective review of bone marrow examination for eight years at lbn Sena Teaching Hospital, mainly to assess the adequacy of the samples as well as to determine the consistency of the bone marrow aspiration findings, the indications for the bone marrow examinations and finally the diagnoses encountered.

\section{MATERIAL AND METHODS}

2008 request forms and reports of bone marrow aspirations carried out from January 2001 to December 2008 at the central hospital laboratory were retrospectively reviewed.

The information extracted included the main indications for performing these procedures:

1. Assessment of cellularity.

2. Identification of focal disease.

3. Lymphoma staging.

the site of sampling, the type of sample(s) obtained, the adequacy of the samples and the diagnoses. The clinical information provided on the request forms as well as the full blood picture findings were also reviewed. The data was analyzed and classified according to the classification of standard text book of hematology classification. ${ }^{8}$

\section{RESULTS}

There were 2008 bone marrow aspirations performed from January 2001 to December 2008 patients' of different age groups. The pediatric age-group was not included. The site of sampling for all patients was the posterior superior iliac crest. There was no documentation of the person who performed the procedure.

patients who had bone trephine biopsies to con firm diagnosis done in private laboratories. the results of biopsies not available, the procedure according to text book of hematology. children

Ann Coll Med Mosul December 2018 Vol. 40 No. 2 
cases not mentioned because no pediatric clinic in In Iben Sena hospital.

All the patients (2008) with bone marrow aspirations were divided into two groups: group1 included 1258 (63\%) patients with non-malignant conditions (Table 1) and group 2 included 750 (37\%) patients with malignant conditions (Table 2).

Table 1. The distribution of nonmalignant conditions in bone marrow aspiration examination.

\begin{tabular}{|c|c|c|c|c|c|c|c|}
\hline 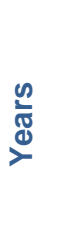 & 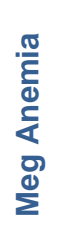 & $\begin{array}{l}\frac{0}{ \pm} \\
\frac{0}{8} \\
\frac{\circ}{0}\end{array}$ & 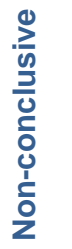 & 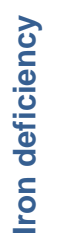 & $\begin{array}{l}\frac{\infty}{d} \\
\frac{\Phi}{\sigma}\end{array}$ & $\begin{array}{l}\overline{\bar{J}} \\
\text { हू } \\
\text { 을 }\end{array}$ & $\begin{array}{l}\bar{\pi} \\
\text { 음 }\end{array}$ \\
\hline 2001 & 7 & 15 & 19 & 19 & 26 & 18 & 104 \\
\hline 2002 & 8 & 4 & 12 & 18 & 25 & 3 & 70 \\
\hline 2003 & 4 & 6 & 8 & 16 & 17 & 18 & 69 \\
\hline 2004 & 11 & 12 & 16 & 45 & 35 & 37 & 156 \\
\hline 2005 & 15 & 5 & 7 & 30 & 52 & 54 & 163 \\
\hline 2006 & 29 & 32 & 54 & 33 & 31 & 74 & 253 \\
\hline 2007 & 21 & 43 & 49 & 33 & 18 & 80 & 244 \\
\hline 2008 & 20 & 37 & 51 & 34 & 9 & 48 & 199 \\
\hline Total & 115 & 154 & 216 & 228 & 213 & 332 & 1258 \\
\hline
\end{tabular}

Notes:\% results from division on 2008

ITP number not mentioned in reports.

Table 2. The distribution of malignant conditions in bone marrow aspiration examination study.

\begin{tabular}{|c|c|c|c|c|c|c|c|}
\hline 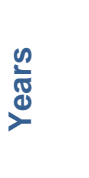 & $\sum_{0}^{+}$ & பل & $\sum$ & $\sum_{<}^{1}$ & $\bar{\alpha}$ & 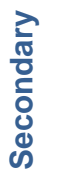 & $\begin{array}{l}\text { 주 } \\
\text { 음 }\end{array}$ \\
\hline 2001 & 1 & 1 & 2 & 13 & 18 & 10 & 45 \\
\hline 2002 & 2 & 1 & 7 & 11 & 5 & 12 & 38 \\
\hline 2003 & 1 & 1 & 3 & 11 & 11 & 16 & 43 \\
\hline 2004 & 2 & 3 & 13 & 18 & 29 & 32 & 97 \\
\hline 2005 & 11 & 6 & 20 & 34 & 31 & 39 & 141 \\
\hline 2006 & 3 & 6 & 9 & 25 & 32 & 64 & 139 \\
\hline 2007 & 5 & 8 & 10 & 25 & 42 & 40 & 130 \\
\hline 2008 & 5 & 7 & 18 & 21 & 29 & 37 & 117 \\
\hline Total & 30 & 33 & 82 & 158 & 197 & 250 & 750 \\
\hline
\end{tabular}

\% results from division on 2008 .
Bone marrow aspirates were performed in this hospital based on clinical grounds and full blood count results.

The non-conclusive bone marrow aspiration samples (216) $11 \%$ were due to the nonavailability of the marrow fragments and dilution of the samples by the peripheral blood.

The others groups (216) $11 \%$ include bone marrow hyperplasia and follow up cases.

\section{DISCUSSION}

Not all patients with hematological malignancies are treated in Ibn Sena hospital; therefore the number of bone marrow aspirates performed is not expected to be large.

The failure rates (inadequate sampling) in this procedure are high (370) $5.4 \%$. It is therefore strongly stressed that maximum effort should be made to obtain BM samples simultaneously with blood regardless of the indications. The inadequate bone marrow aspiration samples due to the non-availability of the marrow fragments and / or dilution by the peripheral blood could also be termed as dry tap. ${ }^{4}$ When the cell trails are diluted, the number of cells available is markedly reduced and hence does not correspond to the cellularity of the fragments, making the morphologic assessment impossible. To overcome this problem, it is suggested that approximately $0.25 \mathrm{ml}$ of marrow is initially aspirated for slide-making, ${ }^{8}$ and a second syringe if required is used to obtain further samples for other tests. ${ }^{9}$

However of more importance is the amount of hemopoietic element in the sample that is available for assessment and diagnosis. It has been shown that the likelihood of detecting a metastatic tumour in a trephine biopsy is an additional. ${ }^{10}$

As this is a retrospective study it is therefore not possible to ascertain the reasons for the failure of obtaining adequate samples for interpretation. Two factors which are known to contribute to poor sampling are faulty techniques ${ }^{11}$ due to inexperienced person performing the procedure and when the bone marrow aspirate is densely cellular or fibrotic. ${ }^{7}$ Many large studies have been conducted to determine the rates of dry taps as percentage of procedures performed. The results ranged from $1.6 \%$ to $6.8 .^{11,12}$ Humphries et al in 1990 reported a rate of $3.9 \%$ from 2,235 simultaneous bone marrow aspirations and 
biopsies. $^{13}$ In other study, 6 out 87 (6.9\%) had a normal finding while the majority had significant bone marrow pathology usually associated with fibrosis, or hypercellularity or both. ${ }^{14}$

In this study, inadequate sampling aspirations were seen in 185 cases, and in 31 (1.72\%) faulty techniques and inadequate reports are likely to be the factors both included in others group. These procedures should be carried out by well-trained individuals who are fully aware of the indications, contraindications and hazards of the procedure. A bone marrow examination should be preceded by clinical evaluation following which a full blood count and a blood film are carried out. A recent full blood picture should be available to be examined with the bone marrow samples as this will not only facilitate interpretation but will also ensure the accurate diagnosis.

Investigation for cases of thrombocytopenia was the most frequent indication for marrow examination and the most frequent diagnosis encountered in this group of patients was immune thrombocytopenic purpura. The indication for bone marrow examination in cases that are clinically suggestive of immune thrombocytopenic purpura is controversial. Jubelirer et al (2002) ${ }^{15}$ suggested that in adults, the procedure is not necessary if a full clinical assessment is made and the hematological laboratory evaluations do not show any other abnormalities. George et $a l^{16}$ also recommended that performing a bone marrow biopsy is unnecessary in patients under the age of 60 years with otherwise normal blood films.

Acute leukaemia was the second most frequent indication and was the most frequent diagnosis encountered in this study.

The yield of adequate marrow sampling for a conclusive diagnosis is present in approximately $50 \%$ in non-malignant cases of BM aspirations. In these cases examination of the trephine biopsy is an indication and therefore a good trephine sampling is essential.

\section{RECOMMENDATIONS}

1. Faulty technique needs to be looked into as a contributing factor to the failure of obtaining good samples.

2. Adequate clinical information with a recent full blood picture should be made available for a more accurate and meaningful interpretation.
3. Communication between the pathologist and the patients' physician is essential to avoid unnecessary bone marrow procedure being done.

\section{CONCLUSIONS}

1. bone marrow examination was done for (1258) $63 \%$ non malignant.

2. Normal bone marrow examination was found to be $(332) 6 \%$.

3. BM examination was done for (750) $7 \%$ Malignant. Acute Leukemia group recognized in (355) $4.8 \%$ as diagnosis and follow up.

Acknowledgements: The researcher wish to thank all Ibn Sina Central Laboratory staff. The authors also thank Dr. Asmma mohammed Ali manager of hematological section, Dr. Bashar Abdulla lecturer of hematology (dept. of pathology) for his suggestions and editorial assistance in the preparation of this manuscript. The authors acknowledge the support provided by the staff of Pathology Department of the college of Medicine, University of Mosul.

\section{REFERENCES}

1.Munker R, Hiller E, Glass J, Paquette J. Modern hematology. 2007. $2^{\text {nd }}$ ed. Humana Press. Totowa, New Jersey,p : 15-19.

2.Brown D, Gutter K. NatkumnumY, Roger W. Bone marrow diagnosis. $20062^{\text {nd }}$ edition. Black well publishing California.p : 5-20.

3.Strauchen JA. The bone marrow biopsy. In: Diagnostic histopathology of the bone marrow. Oxford University Press;1996.p.3-9.

4.Ciesla B. Hematology in practice. 2007. F. A. Davis company. Philadelphia:p. 129-141.

5. Hyun BH, Stevenson AJ, Hanau CA. Fundamentals of bone marrow examination Hematology Oncology Clinics of North America 1994; 8(4): 651- 63.

6.Bain BJ. Bone marrow biopsy morbidity and mortality. Br J Hematol 2003;121:949-51.

7.Bain BJ. Bone marrow trephine biopsy. J Clin Pathol 2001; 54(10): 737-42.

8. Hoffbrand AV, Catavsky D, Tuddenham EGD. Postgraduate hematology. 2005, $5^{\text {th }}$ ed. Blackwell Publishing Ltd, Massachusetts, USA, $p: 1-3$

9. Riley RS. A pathologist's perspective on bone marrow aspiration and biopsy: Performing a bone marrow examination. J Clin Lab Anal. 2004; 18 (2): 70 - 90.

10. Sabwal BD, Malhotra V, Aruna S et al. Comparative evaluation of bone marrow aspirate paticle smears, imprints and biopsy sections. J Postgrad Med 1990; 36(4): 194-8.

11. Bain BJ. Bone marrow aspiration. J Clin Pathol 2001; 54 (9): 657-63.

12. Hoffbrand AV, Pettit JE. Normal haemopoiesis and blood cells. In: Color Atlas of Clinical Haematology, 1998. Mosby-Wolfe, p: 1-34. 
13. Bishop PW, McNally K, Harris M. Audit of bone marrow trephines. J Clin Pathol 1996;49:226-9.

14. Humphries JE. Dry tap bone marrow aspiration: Clinical significant. Am J Hematol 1991;37(3):218-19.

15. Jubelirer SJ. The role of the bone marrow examination in the diagnosis of immune thrombocytopenic purpura: case series and literature review. Clin Appl Thromb Hemost 2001; 8(1): 73-76.
16. George JN, Woolf SH, Raskob GE et al. Idiopathic thrombocytopenic purpura: a practice guideline developed by explicit methods for the American Society of Hematology. Blood. $1996 \mathrm{Jul} ; 88(1): 3-40$. 